\title{
Resolution limit of data-driven coarse- grained models spanning chemical space
}

Cite as: J. Chem. Phys. 151, 164106 (2019); https://doi.org/10.1063/1.5119101

Submitted: 09 July 2019 . Accepted: 01 October 2019. Published Online: 22 October 2019

Kiran H. Kanekal (D), and Tristan Bereau (i)
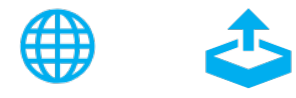

\section{ARTICLES YOU MAY BE INTERESTED IN}

Adventures in DFT by a wavefunction theorist

The Journal of Chemical Physics 151, 160901 (2019); https://doi.org/10.1063/1.5116338

Dual-potential approach for coarse-grained implicit solvent models with accurate, internally consistent energetics and predictive transferability

The Journal of Chemical Physics 151, 164113 (2019); https://doi.org/10.1063/1.5125246

Compatible observable decompositions for coarse-grained representations of real molecular systems

The Journal of Chemical Physics 151, 134115 (2019); https://doi.org/10.1063/1.5116027

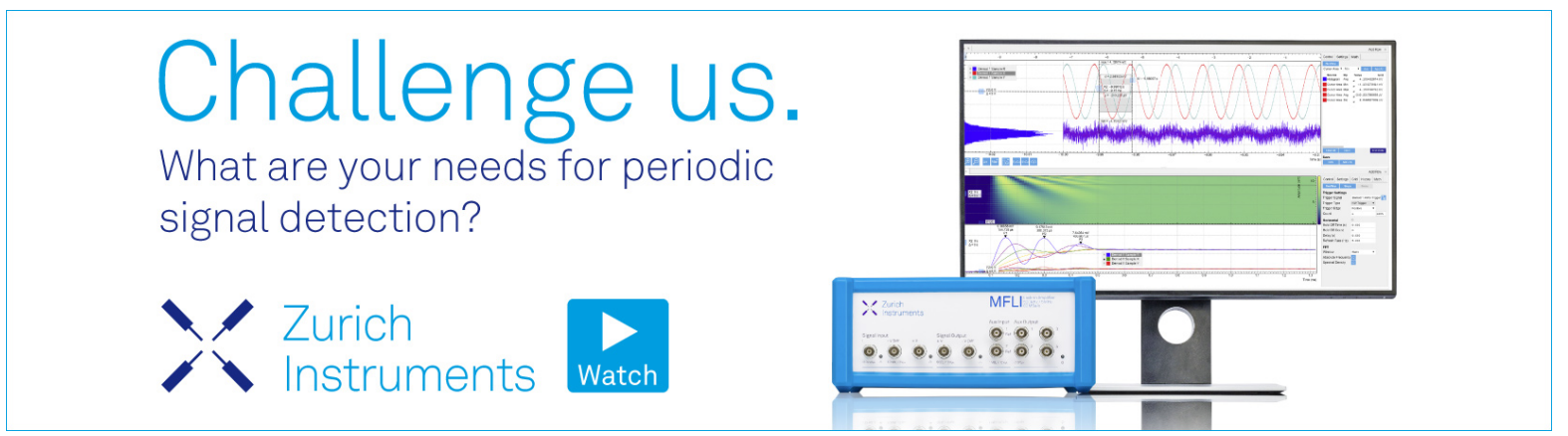




\title{
Resolution limit of data-driven coarse-grained models spanning chemical space
}

\author{
Cite as: J. Chem. Phys. 151, 164106 (2019); doi: 10.1063/1.5119101 \\ Submitted: 9 July 2019 - Accepted: 1 October 2019 • \\ Published Online: 22 October 2019
}

\author{
Kiran H. Kanekal ${ }^{\mathrm{a})}$ (D) and Tristan Bereau
}

\section{AFFILIATIONS}

Max Planck Institute for Polymer Research, Ackermannweg 10, 55128 Mainz, Germany

a) Electronic mail: kanekal@mpip-mainz.mpg.de

\begin{abstract}
Increasing the efficiency of materials design remains a significant challenge given the large size of chemical compound space (CCS). The use of a chemically transferable coarse-grained model enables different molecular fragments to map to the same bead type, significantly increasing screening efficiency. Here, we propose new criteria for the design of coarse-grained models allowing for the optimization of their chemical transferability and evaluate the Martini model within this framework. We further investigate the scope of this transferability by parameterizing three Martini-like models in which the number of bead types ranges from 5 to 16 . These force fields are fully compatible with existing Martini environments because they are parameterized by interpolating the Martini interaction matrix. We then implement a Bayesian approach to determining which chemical groups are likely to be present on fragments corresponding to specific bead types for each model. We demonstrate that a level of accuracy comparable to Martini is obtained with a force field with fewer bead types, using the water/octanol partitioning free energy $\left(\Delta G_{\mathrm{W} \rightarrow \mathrm{Ol}}\right)$ as our metric for comparison. However, the advantage of including more bead types is a reduction of uncertainty when back-mapping these bead types to specific chemistries. Just as reducing the size of the coarse-grained particles leads to a finer mapping of conformational space, increasing the number of bead types yields a finer mapping of CCS. Finally, we note that, due to the large size of fragments mapping to a single Martini bead, a resolution limit arises when using $\Delta G_{\mathrm{W} \rightarrow \mathrm{Ol}}$ as the only descriptor when coarse-graining CCS.
\end{abstract}

Published under license by AIP Publishing. https://doi.org/10.1063/1.5119101

\section{INTRODUCTION}

Molecular design is a cornerstone of materials science, requiring a fundamental understanding of the relationships between the molecular structure and the resulting properties. Traditionally, these structure-property relationships ${ }^{1}$ only arise after multiple rounds of screening and discovery of new materials. ${ }^{2-6}$ These screening approaches constitute examples of direct molecular design in which the space of all chemical compounds, known as chemical compound space (CCS), is explored to determine the most suitable chemistry for the target application. Direct molecular design can be interpreted as projecting a hypersurface in the high-dimensional CCS onto a lower dimensional space defined by certain key molecular descriptors that strongly correlate with the desired property. In contrast, inverse molecular design, in which a structure-property relationship is used to infer a suitable chemical structure from a desired property, remains a "holy grail" of materials science. The main obstacle to achieving this goal is the inability to quickly establish structure-property relationships that can span broad regions of CCS. This is an exceedingly difficult task, given that the size of CCS was estimated to be $10^{60}$ for druglike molecules less than $500 \mathrm{Da}$. Experimentally, this process is inhibited due to both the material and time cost associated with synthesizing and testing a large variety of chemistries that are necessary to infer a relation that is both robust and accurate enough to enable inverse molecular design.

Computationally, recent advancements in processing power and in machine learning have enabled several efficient methods for estimating the electronic properties of a large variety of materials. ${ }^{8-1}$ These methods have the added benefit of screening molecules that cannot be easily synthesized and can thus motivate (or demotivate) the experimental exploration of these chemistries. However, there has been relatively little success in applying computational highthroughput screening methods to determine the stability of chemical 
compounds in soft-matter systems for which thermal fluctuations play a critical role. ${ }^{14,15}$ Force-field based methods, such as molecular dynamics simulations, are typically used to account for the immense number of configurations that result from thermal fluctuations in these systems. Unfortunately, due to the extensive computational resources required, a high-throughput scheme based on atomistic molecular dynamics simulations is currently unfeasible for spanning the large regions of CCS needed to obtain broadly applicable structure-property relationships.

Coarse-grained molecular dynamics simulations provide a means to significantly reduce the computational expense relative to fully atomistic simulations while still capturing the relevant physical properties. ${ }^{16-19}$ Coarse-grained representations of molecules result from mapping groups of atoms to coarse-grained "pseudoatoms" or beads. The governing interactions between beads are determined such that the desired properties of the atomistic system are retained. This usually corresponds to a smoothing of the underlying freeenergy landscape, allowing for more efficient sampling. Conventionally, coarse-graining is applied to a single molecule with the goal of efficiently sampling a specific system of interest. The coarse-grained potentials are obtained via one of several possible methods (e.g., iterative Boltzmann inversion ${ }^{20,21}$ and force-matching ${ }^{22,23}$ ). However, these methods are computationally expensive, requiring an initial atomistic simulation that sufficiently explores the underlying free energy landscape of the system of interest. ${ }^{24}$ Therefore, adapting coarse-grained molecular dynamics simulations to high-throughput screening of chemical compounds requires flexible yet reliable mapping and force field parameterization methods that do not rely on results from higher-resolution simulations for each compound screened.

The coarse-grained Martini force field has become widely used to simulate biological systems as it provides a robust set of transferable force field parameters by constructing biomolecules from a small set of bead types. ${ }^{25-27}$ The Martini model is a top-down model, which maps an atomistic compound or a molecular fragment to a coarse-grained site based on its partitioning between aqueous and hydrophobic environments. ${ }^{28}$ In the context of molecular design, the main advantage that Martini provides is its chemical transferability. While the force field was explicitly parameterized for a set of specific molecules, a single Martini bead can represent several different chemistries that share similar oil/water partitioning characteristics. Thus, the main feature captured by the Martini model is hydrophobicity, which can act as a key driving force in the physics of soft-matter systems. Rather than running a single atomistic simulation that yields a single data point in CCS, a Martini coarse-grained molecular dynamics simulation provides a representative point in CCS, corresponding to the average behavior of all the chemistries that lay in the region surrounding that point. Thus, high-throughput coarse-grained (HTCG) simulations that use chemically transferable force fields, such as Martini, are advantageous because they span vast regions of CCS to quickly infer the structure-property relationships and chemical descriptors that can be used to enable inverse molecular design at any resolution. Menichetti et al. recently demonstrated this by running Martini HTCG simulations to construct a structure-property relationship describing the thermodynamics of the insertion of a small organic molecule into a biological membrane across CCS. ${ }^{29-31}$ In doing so, they discovered a linear relationship between the bulk partitioning behavior of the solute and its potential of mean force. They were then able to identify a structure-property hypersurface to obtain membrane permeabilities for these solute molecules. Using the Generated DataBase (GDB), ${ }^{32,33}$ a systematically computer-generated set of organic druglike compounds, as a proxy for CCS, we then related the regions of this surface to regions of CCS that were dominated by specific chemical moieties, enabling inverse molecular design of small molecules given a desired permeability. The question remains: how representative of CCS is the Martini force field? Given that Martini was designed to reproduce the partitioning behavior of certain solvents as well as the properties of lipid-bilayer membranes, is there a way to accurately parameterize a transferable coarse-grained force field with the goal of optimizing its coverage of CCS? In the context of high-throughput coarse-grained simulations that use Martini, creating a structure-property relationship that enables inverse design requires an understanding of the chemistry that is representative of a specific bead type. The metrics used in assigning specific chemical fragments to Martini bead types mainly consist of several water/oil partitioning free energies, although bulk liquid densities and membrane-specific properties have also been used. ${ }^{28,34}$ Here, we focus specifically on the water/octanol partitioning free energy (although other water/oil partitioning free energies could also be used as they also effectively encode hydrophobicity). Therefore, an intuition for which chemistry maps to a given bead type can only be obtained by understanding how $\Delta G_{\mathrm{W} \rightarrow \mathrm{Ol}}$ varies as a function of chemistry. Given that the number of heavy (nonhydrogen) atoms that usually map to a Martini bead is around four, we can think of each bead as representing a small carbon scaffold perturbed to some degree by either replacing carbons with other heavy atom types (e.g., oxygen, nitrogen, or fluorine) or by replacing single bonds with double or triple bonds. We define a functional group as being one or a localized combination of these types of perturbations.

In this work, we quantify the information loss that occurs when a top-down coarse-grained model, like Martini, is used to reduce the resolution of CCS. Additionally, we parameterize three sets of coarse-grained force fields in the Martini framework. In this context, we use the terms "force field" and "model" interchangeably, defined as a set of parameters which describe the interactions between a fixed number of coarse-grained representations called bead types. Each force field developed in this work consists of 5,9 , and 16 neutral bead types, as well as two extra types to account for hydrogen-bond donors and acceptors. We observe that Martini does not provide the most efficient reduction of CCS. We show that the nine-bead force field reduces CCS to the same degree as Martini despite having three fewer bead types and that further increasing the number of bead types yields negligible improvements in the performance of the model. The models are validated by performing coarse-grained simulations to calculate the water/octanol partitioning free energies of approximately 500 compounds for which experimental data are available. Finally, we demonstrate that the main advantage of a force field with a large number of bead types is the reduction of uncertainty when back-mapping these coarsegrained representations to real chemical functional groups. Just as decreasing the resolution of the coarse-grained mapping reduces the resolution of the potential energy landscape, a reduction in the number of bead types of a chemically transferable coarse-grained force field allows for an increased degeneracy of chemical fragments 


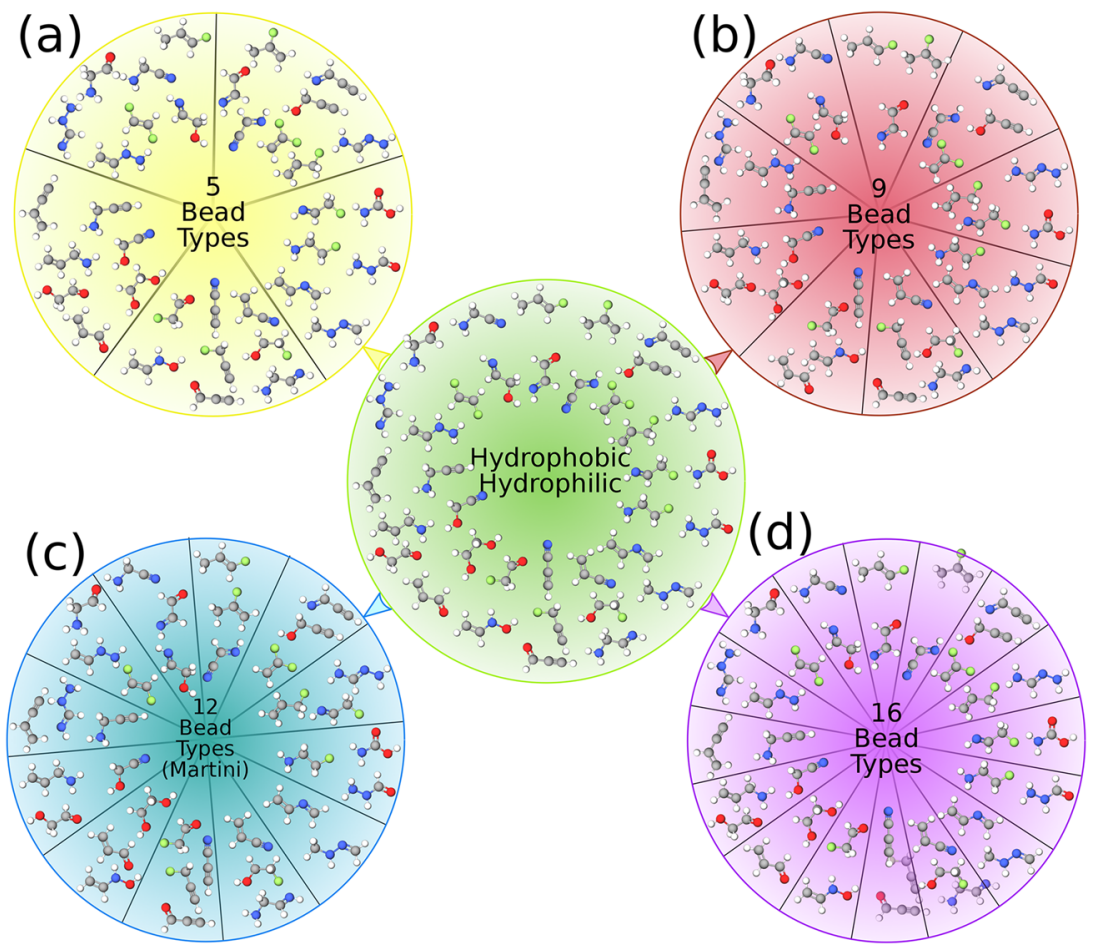

FIG. 1. A cartoon schematic showing the projection of CCS onto the hydrophobicity descriptor $\Delta G_{W \rightarrow O}$, allowing for the creation of top-down chemically transferable coarse-grained models with (a) five, (b) nine, (c) 12, and (d) 16 bead types. The number of bead types included in these models defines the degree to which CCS is partitioned on the $\Delta G_{W \rightarrow O}$ axis. By varying the number of bead types in each model, we obtain greater insight into the range of chemistries spanned by a single bead type.

that map to a single bead type, illustrated in Fig. 1. Ideally, a welldesigned chemically transferable coarse-grained force field would contain some number of bead types that can be intuitively backmapped to single chemical functional groups. However, the size of a single functional group is small relative to the size of a Martini bead such that many functional groups could be identified within a fragment mapping to a single Martini bead. Here, we demonstrate that this mismatch between the size of a Martini bead and a single functional group requires additional constraints in order to identify the unique chemistry that maps to each bead type. Incorporating these constraints into a Bayesian formalism yields probabilities of specific chemistries mapping to a given bead type, further promoting inverse molecular design. However, even these additional constraints allow for the same functional groups to be present in multiple bead types, indicating a natural resolution limit when using $\Delta G_{\mathrm{W} \rightarrow \mathrm{Ol}}$ as the sole basis for a chemically transferable, top-down coarse-grained model.

\section{METHODS}

\section{A. The auto-Martini algorithm}

This work relies on the AUTO-MARTINI algorithm initially developed by Bereau and Kremer. ${ }^{35}$ The algorithm first determines an optimal mapping for an organic small molecule. The mapping provides the number of coarse-grained beads used to represent the molecule as well as their placement. A mapping cost function is minimized for each molecule so as to optimize both the number and placement of beads used in its coarse-grained representation. The assignment of coarse-grained potentials to each bead (bead-typing) occurs by assigning an existing Martini bead type that has the closest matched water/octanol partitioning free energy $\left(\Delta G_{\mathrm{W} \rightarrow \mathrm{Ol}}\right)$ with that of the molecular fragment encapsulated by the bead. The partition coefficients of these fragments are obtained by using ALOGPS, ${ }^{36,37}$ a neural network algorithm that predicts these values given the chemical structure of the fragment. In this work, we use an updated version of the AUTO-MARTINI algorithm that has three significant changes from the previous version. The first change is an increased energetic penalty for "lonely" atoms (i.e., atoms that fall outside the van der Waals radius of the placed coarse-grained beads). The second change is a reduction of the multiplicative factor used when assigning bead types to rings for both five and six-membered rings. Finally, the cutoff value for the $\Delta G_{\mathrm{W} \rightarrow \mathrm{Ol}}$ for the assignment of donor and acceptor fragments to their corresponding bead types was modified such that the coarse-grained and atomistic population distributions more closely matched. All of these changes increased the algorithm's accuracy, which is quantified in the supplementary material (Sec. II). The updated code is available for use via a GITHUB repository. Using the refined AUTO-MARTINI algorithm, approximately $3.5 \times 10^{6}$ molecules with ten heavy atoms or less that make up the GDB were mapped to coarse-grained representations for four different force fields. The molecules contain carbon, nitrogen, oxygen, fluorine, and hydrogen atoms only. Of these $3.5 \times 10^{6}$ compounds, approximately 340000 were successfully mapped to both coarse-grained unimers ( 1 bead representations) and dimers ( 2 bead representations) for all of the force fields described in this work. The majority of the remaining compounds were mapped to coarse-grained representations with a higher number of beads, and a small fraction 
of compounds were unable to be successfully mapped by the algorithm. Histograms comparing the distributions of $\Delta G_{\mathrm{W} \rightarrow \mathrm{Ol}}$ for each set of atomistic compounds mapping to coarse-grained unimers and dimers and their coarse-grained counterparts were constructed using the NUMPY histogram function, ${ }^{39}$ with the number of bins being equal to 1000 and 1050 for unimers and dimers, respectively. These histograms are shown in Figs. 2(a)-2(d) for Martini, while the other histograms can be found in the supplementary material (Fig. S4). Here, we noticed two systematic errors of the prescribed Martini
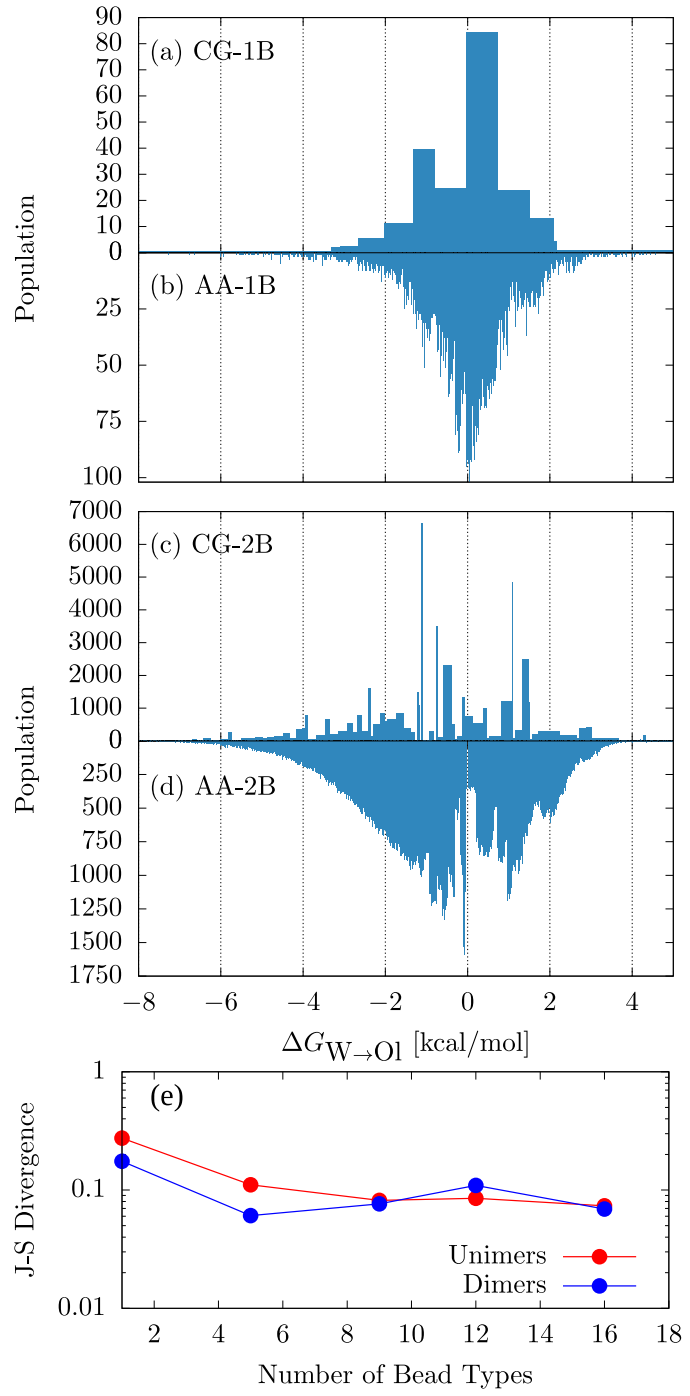

FIG. 2. Histograms of 343700 small molecules extracted from GDB that map onto one-bead or two-bead coarse-grained Martini representations. [(a) and (c)] Coarse-grained and [(b) and (d)] atomistic populations as a function of water/octanol partitioning free energy. The width of the bars in (a) and (c) corresponds to the range of atomistic water/octanol partitioning free energies that can map to that coarse-grained representation. (e) Jensen-Shannon divergence calculated for the histograms corresponding to those in (a)-(d) for all force fields described in this work. mapping process that are done by AUTO-MARTINI. About $14 \%$ of the GDB molecules were undermapped by the algorithm, meaning that molecules and fragments with greater than seven heavy atoms were mapped to single Martini beads. These molecules are included in the histograms but do not significantly affect the results of various statistical analyses conducted in this work. The other error is the treatment of molecules containing five-membered rings, which make up roughly $18 \%$ of the dataset obtained using AUTO-MARTINI. AUTO-MARTINI assigns two "small" beads to represent these rings. While this does technically follow the mapping rule for these "small" beads (which should map two to three heavy atoms to a bead), ring planarity is no longer enforced with only two beads. Overall, these systematic errors have a negligible impact on our findings, and we include further discussion of these issues in the supplementary material (Sec. III).

\section{B. The Jensen-Shannon divergence}

In this work, the main tool used to quantify information loss when going from atomistic to coarse-grained resolution is the relative entropy in the form of a Jensen-Shannon divergence (JSD or $\left.D_{\text {IS }}\right) .{ }^{40}$ The relative entropy framework has been previously established as a useful tool for evaluating the quality of coarsegrained models. ${ }^{41,42}$ The JSD is a variation of the well-known Kullback-Leibler divergence ${ }^{43}\left(D_{\mathrm{KL}}\right)$ used to calculate the relative entropy between two distributions. It offers two advantages over the Kullback-Leibler divergence in that it is symmetric and always has a finite value. Rather than directly relating two distributions, as is the case for the Kullback-Leibler divergence, the JSD computes the relative entropy by comparing each of these distributions to a third distribution which is the average of the other two distributions, as shown in the following equations:

$$
\begin{gathered}
D_{J S}=\frac{1}{2} D_{K L}\left(P_{\mathrm{CG}} \| P_{\mathrm{avg}}\right)+\frac{1}{2} D_{K L}\left(P_{A A} \| P_{\text {avg }}\right), \\
\text { where } D_{K L}(A \| B)=\sum_{i=1}^{N} a_{i} \ln \left(\frac{a_{i}}{b_{i}}\right) \\
\text { and } P_{\text {avg }}=\frac{1}{2}\left(P_{C G}+P_{A A}\right) .
\end{gathered}
$$

In the above equations, we define $D_{K L}$ in terms of two arbitrary distributions, $A$ and $B$ with $N$ elements $a_{i}$ and $b_{i}$. Here, we use the JSD to evaluate how well the distribution of the water/octanol partitioning free energies for the coarse-grained molecules $\left(P_{C G}\right)$ matches the corresponding distribution at the atomistic resolution $\left(P_{A A}\right)$. A value of 0 indicates that the two distributions are the same. The use of the average distribution $\left(P_{\text {avg }}\right)$ conveniently prevents divisions by zero when comparing histograms like those shown in Figs. 2(a)-2(d).

\section{Basin-hopping and minimization schemes}

In this work, we use multiple methods to optimize the coarsegrained partitioning free energies to best match the atomistic distribution of free energies. The first such method is the basin-hopping method, ${ }^{44}$ which is a variation of Metropolis-Hastings Monte Carlo. The algorithm proceeds in the following steps. Given a set of initial coordinates and objective function, the initial coordinates are first 
randomly perturbed and subsequently minimized. The results of the minimization are either accepted or rejected based on a predefined Metropolis criterion. These two steps form a single iteration of the algorithm, and a large number of iterations may be required to find the desired minima. Here, we use the JSD as our objective function and a set of possible water/octanol partitioning free energies for each coarse-grained bead type as our initial coordinates. Each move then corresponds to shifting the values of $\Delta G_{\mathrm{W} \rightarrow \mathrm{Ol}}$ for each coarsegrained bead type in a given force field. The optimizations were performed in order to define the desired $\Delta G_{\mathrm{W} \rightarrow \mathrm{Ol}}$ values for the fivebead-type force field, using the BASINHOPPING function provided by SCIPY $^{45}$ with a Broyden-Fletcher-Goldfarb-Shanno local minimizer, ${ }^{4}$ a Metropolis temperature parameter of 0.008 , and a step size of $0.024 \mathrm{kcal} / \mathrm{mol}$. For the reference atomistic distribution, we applied the ALOGPS neural network to predict $\Delta G_{\mathrm{W} \rightarrow \mathrm{Ol}}$ for all molecules in the GDB with eight heavy atoms or less that were known to map to single bead Martini representations using the AUTO-MARTINI algorithm. However, finding the optimal set of $\Delta G_{\mathrm{W} \rightarrow \mathrm{Ol}}$ values for the 16-bead-type force field using this approach proved to be computationally unfeasible, as the dimensionality of the problem scales with $M^{N}$, where $N$ is the total number of bead types in the force field and $M$ is the range of $\Delta G_{\mathrm{W} \rightarrow \mathrm{Ol}}$ values spanned by the Martini bead types divided by the step size. To parameterize the 16-bead-type force field, we used the SCIPY minimize function ${ }^{45}$ with the modified Powell method, ${ }^{46}$ starting with an initial set of 18 bead types that were evenly distributed along the $\Delta G_{\mathrm{W} \rightarrow \mathrm{Ol}}$ axis. The results of the minimization indicated two sets of two bead types that were within $0.1 \mathrm{kcal} / \mathrm{mol}$ of each other, and so each pair was combined into a single bead type, resulting in 16 bead types total.

\section{Clustering the GDB}

In addition to the optimization of the JSD, a new set of coarsegrained water/octanol partitioning free energies was also proposed by clustering the GDB, leading to the 9-bead-type force field. Specifically, all GDB molecules with eight heavy atoms or less were grouped based on the number and type of heteroatom substitutions present in the molecule (i.e., the number of times that a $\mathrm{C}$ was replaced with $\mathrm{N}, \mathrm{O}$, or $\mathrm{F}$ ). The resulting atomistic molecular populations as well as the mean and standard deviation of their water/octanol partitioning free energies are shown in Fig. 3. Detailed information on each of the distributions (beyond what is provided in Fig. 3) is available in the supplementary material (Sec. V and the ZENODO ${ }^{47}$ repository). The desired water/octanol partitioning free energies are determined by clustering the points on this graph, starting from the highest populated points and accepting anything that was within $\pm 0.5 \mathrm{kcal} / \mathrm{mol}$ of these points. For example, the first point with the highest population in Fig. 3(a) is chosen as a starting point for the first bead type. All points that fall within $0.5 \mathrm{kcal} / \mathrm{mol}$ are assigned to this bead type, and the $\Delta G_{\mathrm{W} \rightarrow \mathrm{Ol}}$ is determined by taking a population-weighted average of all of these points. The next bead type is determined by selecting the highest point in Fig. 3(a) that is not already assigned to a bead type and repeating the process. For both this clustering and for the numerical optimization methods discussed in Sec. II C, the maximum number of heavy atoms per molecule was limited to eight. For all other data-driven calculations, all GDB molecules with up to ten heavy atoms were included.

\section{E. Functional group analysis}

A statistical analysis of the functional groups found in the molecular fragments mapping to single beads is necessary in order to obtain a more detailed picture as to which chemistries are representative of specific bead types. The enumeration of functional groups was achieved through the use of the CHескмоL software developed by Haider. ${ }^{48}$ This software uses the $3 \mathrm{D}$ coordinates of each atom and the corresponding atom labels in a given molecule to identify
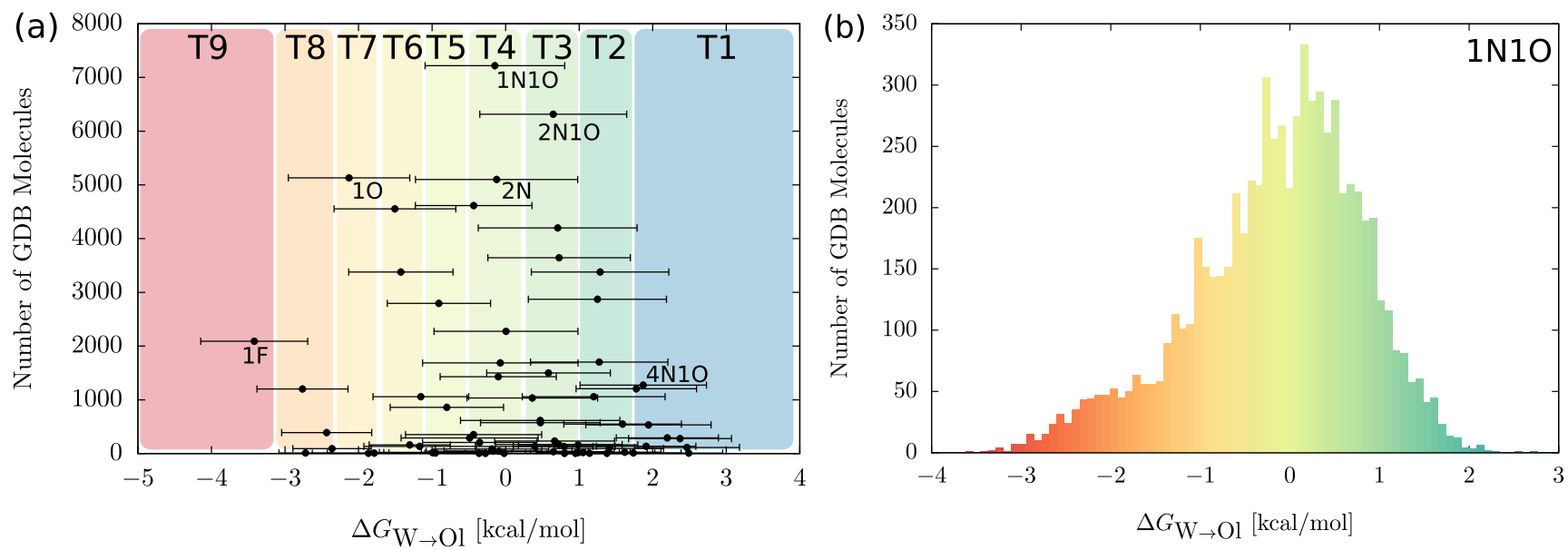

FIG. 3. (a) Population vs average values of the distributions of water/octanol partitioning free energies corresponding to GDB molecules with up to 8 heavy atoms and a specific number and type of heteroatom substitutions. The error bars refer to the standard deviations of each distribution. The colored backgrounds denote how these average values are clustered to obtain new bead types that more efficiently divide CCS. (b) Example distribution corresponding to top-most point labeled in the graph on the left. The label refers to the number and type of heteroatom substitution, in this case 1 nitrogen and 1 oxygen substitution. The color applied to the histogram corresponds to the colors shown in (a), indicating the bead types to which these molecules would be assigned. 
common chemical functional groups. A full list of the functional groups identified can be found in the supplementary material (Sec. VII and the ZENODO ${ }^{47}$ repository). Using CHECKMOL, we determine the degeneracy of specific functional-group pairs with respect to single bead types for the set of molecular fragments that mapped to a single bead. This amounts to counting the number of fragments containing a specific functional group pair and mapping to a single bead type. This population is then normalized with respect to the total number of fragments containing the same functional group pair across all bead types. It is useful to frame this statistical analysis
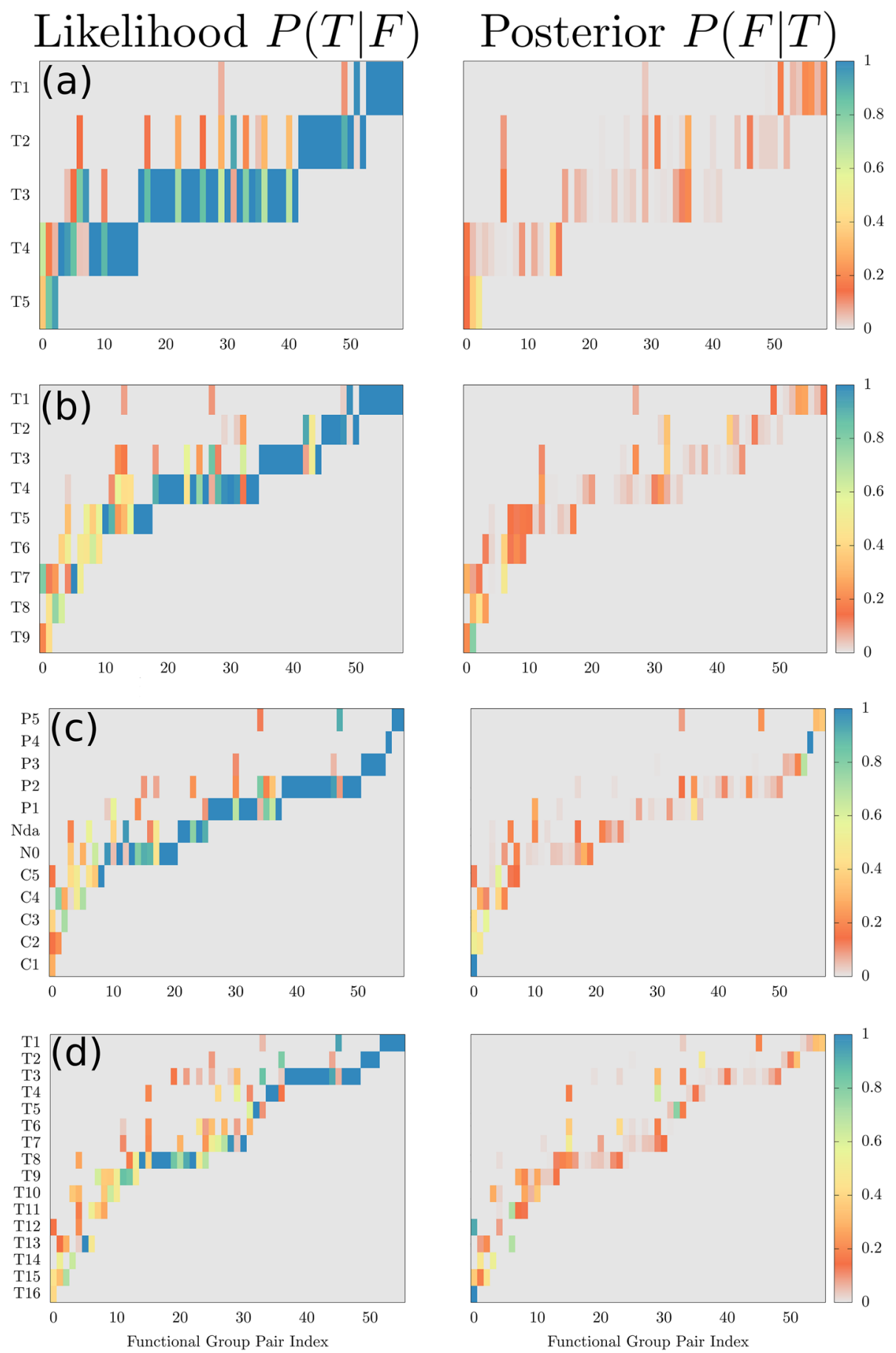

FIG. 4. Heat maps portraying the degeneracy of specific pairs of functional groups for a given bead type for force fields containing five (a), nine (b), 12 (c), or 16 (d) bead types. The horizontal axes denote specific functionalgroup pairs that exist in a chemical fragment with five heavy atoms only. The color corresponds to either the columnnormalized or row-normalized probabilities. The column-normalized probabilities (left side) are equivalent to the Bayesian likelihood of a given functional group mapping to a specific bead type. The row-normalized heat maps (right side) show the Bayesian posterior probabilities of obtaining a specific functional group given a bead type. 
in terms of conditional probabilities, as this yields specific information relevant for molecular-design applications. For example, the aforementioned counting and normalization is equivalent to calculating the likelihood of assigning a bead type $(T)$ given a specific functional group pair $(F)$, defined as $P(T \mid F)$. We use the fragment population distributions for each bead type and each functional group pair to obtain probabilities $P(T)$ of a bead type and $P(F)$ of a functional group pair. We then calculate the posterior probabilities $P(F \mid T)$ of a given bead type back-mapping to a specific functional group pair using Bayes' theorem

$$
P(F \mid T)=\frac{P(T \mid F) P(F)}{P(T)} .
$$

The results are shown as a series of heat maps for each force field in Fig. 4.

\section{F. Parameterization of new bead types}

The new force fields share most of the parameters defined by the Martini force field. ${ }^{34}$ For the intramolecular interactions, bonded, angle, and dihedral force constants remain the same as those prescribed by Martini. The nonbonded interactions only deviate from Martini through the strength of the potential used. We linearly interpolate across the interaction matrix defined in Martini, ${ }^{34}$ utilizing the distance between the established Martini $\Delta G_{\mathrm{W} \rightarrow \mathrm{Ol}}{ }^{35}$ and the desired $\Delta G_{\mathrm{W} \rightarrow \mathrm{Ol}}$ for the interpolation. The partitioning free energies of each bead type were then confirmed by running coarse-grained molecular dynamics simulations of single beads of each new bead type. These results are included in the supplementary material (Sec. VI) and show that this method yields an accurate force field without relying on an iterative scheme. Linear interpolation is chosen as it is clear that there is no underlying functional form or smooth landscape that can be derived from this parameter space (see supplementary material, Sec. VI for details). The new bead types are named $\mathrm{T} i$ types, with $i$ ranging from 1 to $N$, where $N$ is the total number of bead types in the force field. The numbering is also ordered by polarity. For example, the T1 bead type for all new force fields is the most polar type. Conversely, the T5, T9, and T16 bead types are the most apolar bead types in the 5, 9, and 16-bead-type force fields, respectively. The full list of bead types for each force field, their force field parameters, and their corresponding $\Delta G_{\mathrm{W} \rightarrow \mathrm{Ol}}$ values is available in the supplementary material (Sec. VI and the ZENODO ${ }^{47}$ repository).

\section{G. Coarse-grained simulations}

Coarse-grained molecular dynamics simulations were performed in GROMACs ${ }^{49}$ version 4.6.6 using the standard Martini force field parameters ${ }^{34}$ as well as the new force field parameters derived in this work. A time step of $\delta t=0.03 \tau$ was used for all simulations, where $\tau$ is the natural time unit for the propagation of the model defined in terms of the units of energy $\mathscr{E}$, mass $\mathscr{M}$, and length $\mathscr{L}$ as $\tau=\mathscr{L} \sqrt{\mathscr{M} / \mathscr{E}}$. The simulations were run in an NPT ensemble with a Langevin thermostat and Andersen barostat $^{50}$ to keep the temperature and pressure at $300 \mathrm{~K}$ and 1 bar, respectively. The corresponding coupling constants were $\tau_{T}=\tau$ and $\tau_{P}=12 \tau$
Water/octanol partitioning free energies were obtained by simulating approximately 500 coarse-grained molecules in octanol and water. Approximately, 250 octanol molecules and 350 Martini water molecules were simulated for their respective systems, with the appropriate number of antifreeze particles. ${ }^{34}$ The free energies were computed using the Bennett acceptance ratio method ${ }^{51}$ in which the coarse-grained solute was incrementally decoupled from the solvent via the coupling parameter, $\lambda .21$ simulations were run for each molecule at evenly spaced $\lambda$ values ranging from 0 to 1 , with each simulation run for 200000 time steps. Finally, the partitioning free energies were calculated using the relation $\Delta G_{\mathrm{W} \rightarrow \mathrm{Ol}}=\Delta G_{\mathrm{Ol}}-\Delta G_{\mathrm{W}}$. As this method does not take into account the saturation of the octanol phase with water that occurs in experimental systems, ${ }^{52}$ we also ran some test simulations using an octanol phase which contained 25\% molar water molecules. We found that only coarse-grained molecules containing a majority of highly polar beads would show a reduction in their $\Delta G_{\mathrm{W} \rightarrow \mathrm{Ol}}$ values due to increased contact with water in the water-saturated octanol phase (Fig. S5 in the supplementary material).

\section{RESULTS}

\section{A. Quantifying information loss of coarse-grained models with varying number of bead types}

The updated AUTO-MARTINI algorithm was used to first map and subsequently assign bead types to $3.5 \times 10^{6}$ molecules of the GDB containing ten or fewer heavy atoms using the Martini force field as well as the other three force fields parameterized by interpolating the Martini interaction matrix. Figure 2 shows a comparison of the atomistic and coarse-grained $\Delta G_{\mathrm{W} \rightarrow \mathrm{Ol}}$ distributions for molecules mapping to Martini unimers [Figs. 2(a) and 2(b)] and dimers [Figs. 2(c) and 2(d)]. The corresponding histograms for the other three force fields as well as a histogram constructed using the Martini force field but with the original AUTO-MARTINI algorithm can be found in the supplementary material (Fig. S4). The width of the coarse-grained bars reflects the range of $\Delta G_{\mathrm{W} \rightarrow \mathrm{Ol}}$ values within which a molecule must fall in order to be assigned that bead type, or, in the dimer case, a combination of bead types. The height of the bars is set such that the area covered by each bar is equal to the total number of molecules that were assigned that coarse-grained representation. We then calculate the JSD between the coarse-grained and atomistic histograms for each force field to quantify the information loss as a function of the number of bead types present in each force field [Fig. 2(e)]. Increasing the number of bead types reduces the information loss when going from atomistic to coarse-grained resolution, though this reduction becomes insignificant after reaching nine bead types. The JSD comparing the unimer histograms [red curve in Fig. 2(e)] changes negligibly when increasing the number of bead types from 9 to 16, with only a small increase for the Martini case (12 bead types). This is expected due to the fact that the atomistic histogram of GDB molecules mapping to a single bead is a simple, unimodal distribution with a peak at $\Delta G_{\mathrm{W} \rightarrow \mathrm{Ol}}=0$. Since all of the force fields have at least one amphiphilic bead type with a $\Delta G_{\mathrm{W} \rightarrow \mathrm{Ol}}$ close to 0 , they all capture this defining feature of the histogram, and, comparatively, further information gains are negligible. Remarkably, we find the JSDs were 
largely insensitive to the choice of numerical optimization technique used for the derivation of each force field, as they all capture this prominent feature.

The ISDs calculated from the dimer histograms [blue curve in Fig. 2(e)] show a variety of interesting features. Both the 9 and 16-bead-type force fields maintain roughly the same JSD, suggesting that the combinatorial explosion that results from doubling the molecular weight is captured by these force fields. The slight increase seen in the unimer JSD for Martini is noticeably amplified for the dimer case, indicating that careful placement of bead types on the $\Delta G_{\mathrm{W} \rightarrow \mathrm{Ol}}$ axis is necessary to maximize chemical transferability. Surprisingly, the greatest deviation in the JSD going from the unimer to dimer histogram comes from the fivebead-type force field, dropping well below the values for the higher bead type force fields. The reason for this is shown in Fig. S3b, which shows that the distribution of atomistic compounds mapping to dimers in the five-bead-type force field is significantly different from its analogs for the other force fields. This indicates that a significant number of molecules that would map to dimers when using one of the other force fields are mapped to trimers or tetramers using the 5-bead-type force field. Unfortunately, we were unable to compute histograms of molecules corresponding to coarse-grained trimers or tetramers due to computational constraints: in order to get a converged distribution that could represent the chemical space corresponding to molecules mapping to trimers, we would need to run the AUTO-MARTINI algorithm on the GDB molecules containing up to at least 15 heavy atoms (assuming a 5 heavy atom to 1 bead mapping), which is computationally unfeasible due to the exponential growth of CCS as a function of molecule size.

\section{B. Relating chemistry to bead types}

As an alternative to purely numerical methods for determining the optimal $\Delta G_{\mathrm{W} \rightarrow \mathrm{Ol}}$ values for the bead types of a coarse-grained force field that best partitions CCS, we cluster the GDB itself and use the weighted average of $\Delta G_{\mathrm{W} \rightarrow \mathrm{Ol}}$ for each cluster. Figure 3(a) shows the two descriptors upon which we project and subsequently cluster the GDB. Each point in Fig. 3(a) represents the set of molecules in the GDB that have a specific number and type of heavy atom substitutions (i.e., $\mathrm{N}, \mathrm{O}$, or F). The points are placed on the $\Delta G_{\mathrm{W} \rightarrow \mathrm{Ol}}$ axis according to the average of their $\Delta G_{\mathrm{W} \rightarrow \mathrm{Ol}}$ distribution. The error bars represent the standard deviation of the $\Delta G_{\mathrm{W} \rightarrow \mathrm{Ol}}$ in each distribution. One of the corresponding distributions is shown in Fig. 3(b), with the rest available in the zENODO repository. ${ }^{47}$ The points are clustered hierarchically with respect to population and average as shown in Fig. 3(a). The highest-populated points are all chosen as cluster centers as long as they are separated by at least $0.5 \mathrm{kcal} / \mathrm{mol}$, which is an arbitrarily chosen length-scale for the clustering to ensure a reasonable number of bead types in the final force field. After the points are clustered, the desired $\Delta G_{\mathrm{W} \rightarrow \mathrm{Ol}}$ of each bead type is determined by taking the population-weighted average of all the points in a cluster. This intuitively provides a basic understanding of the chemistry that maps to a specific bead type. For example, a T4 bead is more likely to back-map to a molecule with one $\mathrm{N}$ and one $\mathrm{O}$ substitution compared to two $\mathrm{N}$ substitutions because of the difference in the GDB populations of each molecule type.
It is important to characterize the degree to which unique chemistries are captured by the bead types of each force field. Using the GDB as a proxy for CCS enables a quantitative understanding of the chemical transferability of each bead type through the calculation of conditional probabilities. Figure 4 shows a series of heat maps corresponding to each of the four force fields investigated in this work. These heat maps are constructed by counting all fragments containing only five heavy atoms and assigned to a specific bead type such that two functional groups are detected by the CHECKMOL software package. The fragment population distributions are then used to calculate the Bayesian likelihood $P(T \mid F)$ and posterior $P(F \mid T)$ for each bead type/functional pair combination in every force field. The numbers on the horizontal axis for each heat map denote specific pairs of functional groups found in the chemical fragments that are assigned to a bead type, while the color corresponds to either the likelihood or posterior probabilities. We see the localization of functional-group pairs to specific bead types mainly because of the constraint of only including fragments with five heavy atoms. This constraint limits the combinatorics of heteroatom and bond substitutions that result in functional-group pairs. Despite the addition of these constraints, a large number of functional-group pairs are still split across multiple bead types. The corresponding heat maps constructed using four-heavy-atom fragments only are included in the supplementary material (Sec. VII) and show far less degeneracy of functional-group pairs across bead types compared to these heat maps, although the general trends observed are the same. Table I provides additional quantification of the trends shown in Fig. 4, displaying the average number of functional-group pairs per bead type, as well as the number of likelihood and posterior values above cutoff values of 0.99 and 0.2 , respectively. As the number of bead types increases, both the average number of functional-group pairs per bead type and the number of likelihood values greater than 0.99 decrease, indicating that fewer bead types in a force field increase the coverage of CCS for each bead type. The opposite trend is observed for the number of posteriors greater than 0.2 , indicating that more bead types result in higher chemical specificity for each bead type.

\section{Coarse-grained force field validation}

While we have demonstrated that the careful placement of bead types on the $\Delta G_{\mathrm{W} \rightarrow \mathrm{Ol}}$ axis leads to more chemical transferability, the force fields themselves must be validated. Because $\Delta G_{\mathrm{W} \rightarrow \mathrm{Ol}}$ was used as the target property for the interpolation of the Martini

TABLE I. For each force field, the number of bead types, the average number of functional-group pairs per bead type, the total number of likelihood values over 0.99 , and the total number of posterior values over 0.2 .

\begin{tabular}{lccc}
\hline \hline $\begin{array}{l}\text { No. of bead } \\
\text { types }\end{array}$ & $\begin{array}{c}\text { Avg. no. of } \\
\text { func. group pairs }\end{array}$ & $\begin{array}{c}\text { No. of } \\
\text { likelihoods }>0.99\end{array}$ & $\begin{array}{c}\text { No. of } \\
\text { posteriors }>0.20\end{array}$ \\
\hline 5 & 16.4 & 40 & 8 \\
9 & 10.1 & 33 & 17 \\
12 (Martini) & 7.4 & 35 & 20 \\
16 & 6.4 & 27 & 26 \\
\hline \hline
\end{tabular}


interaction matrix, we must ensure that this property is indeed captured by the resulting models and determine to what extent the accuracy of these models changes as the number of bead types increases. Because we used the Martini interpolation matrix in our parameterization, our force fields are, by construction, fully compatible with the existing Martini model. This allows us to use the Martini solvent models for both water and octanol in our validation, without having to derive new solvent models for each new force field. Essentially, this means that, using the methods outlined in this work, it is possible to parameterize any number of new bead types with desired $\Delta G_{\mathrm{W} \rightarrow \mathrm{Ol}}$ values within the $\Delta G_{\mathrm{W} \rightarrow \mathrm{Ol}}$ range covered by Martini that will also be compatible with the Martini model. Figure 5 shows correlation plots comparing $\Delta G_{\mathrm{W} \rightarrow \mathrm{Ol}}$ values computed from coarsegrained MD simulations with experimental values for approximately 500 ring-less molecules obtained from the National Cancer Institute database. ${ }^{53}$ The comparison is made for all four of the models examined in this work. The number of compounds varies for each model, as the AUTO-MARTINI algorithm was able to successfully find mappings for more molecules in the database when using a model with a higher number of bead types, ranging from 479 compounds mapped when using the 5-bead-type model to 505 when using the 16-beadtype model. The full set of compounds as well as their corresponding coarse-grained representations is provided in the supplementary material (specifically in the zENODO ${ }^{47}$ repository). The vertical series of points prominently shown in Fig. 5(a) are a consequence of the increased degeneracy of CCS for the 5-bead-type model: they represent many compounds mapping to the same coarse-grained representation. As expected, the correlation becomes less discretized as the number of bead types increases. Examining Figs. 5(e) and 5(f), we see corresponding gains and losses in the Pearson correlation coefficients and mean absolute errors (MAEs), respectively. Surprisingly, the gains in accuracy are very slight as a function of number of bead types-with the correlation coefficient only increasing by 0.01 and the MAE decreasing by $0.2 \mathrm{kcal} / \mathrm{mol}$-despite tripling the number of bead types. Even with the five-bead-type model, we achieve an MAE of $0.8 \mathrm{kcal} / \mathrm{mol}$, within the standard for chemical accuracy. We deliberately chose not to include molecules that contained rings because this version of the Martini force field quantitatively fails in modeling molecular rings for many documented reasons. These reasons include lack of cross-parameterizations between
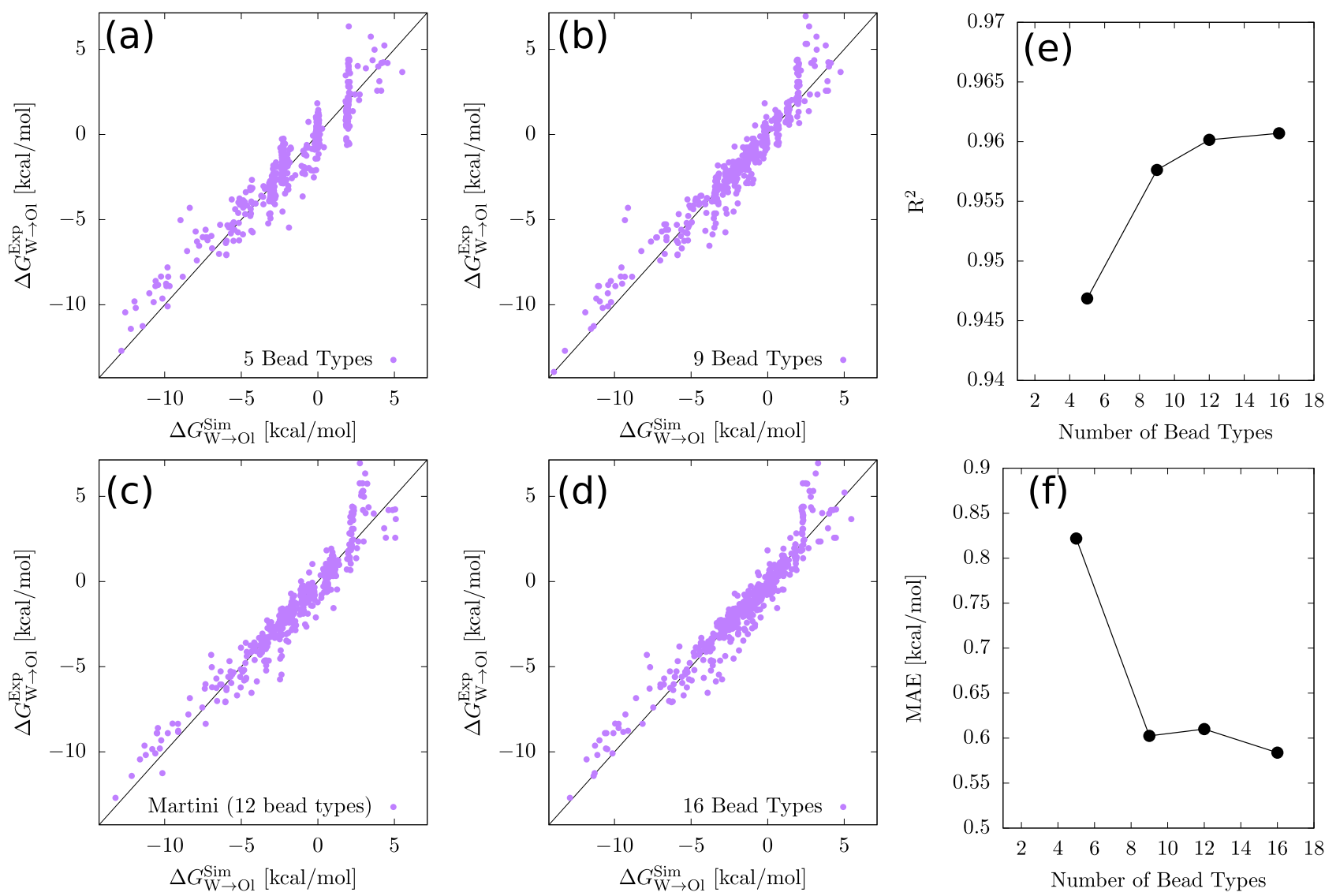

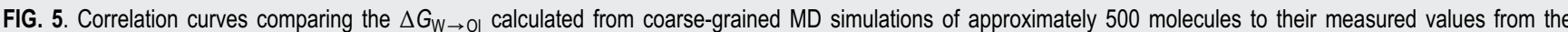
experiment. The results are shown for each of the force fields described in this work [(a)-(d)] as well as their respective Pearson correlation coefficients and mean absolute error (MAE) values [(e) and (f)]. 
normal-sized and the "small" sized beads used to model rings, ${ }^{54}$ as well as the size disparity between the atomistic and coarse-grained ring structures. ${ }^{55}$ For these reasons, and in anticipation of the new Martini version 3.0 that is currently being developed to address these flaws, we refrained from addressing ring molecules in this study.

\section{DISCUSSION}

Given the immense size of CCS, the creation of reduced models that efficiently subdivide the space is necessary for screening applications. Here, we demonstrate the use of the water/octanol partitioning free energy as the parameter used to generate top-down chemically transferable coarse-grained models of varying numbers of bead types. This choice of descriptor is inspired by the Martini force field, which prescribes the use of partitioning free energies as the tool to encode the natural hydrophobic/hydrophilic character of a molecule when determining the bead type to be used to represent a molecular fragment. ${ }^{28}$ A major strength of Martini is its ability to model this important molecular property using simple LennardJones potentials with varying attractive well depths. Here, we use the GDB as a proxy for $\mathrm{CCS}^{29,31}$ and apply the AUTO-MARTINI algorithm to compare the populations of the GDB molecules and their corresponding coarse-grained representations for four different force fields with varying numbers of bead types. This effectively amounts to a discretization of CCS projected onto $\Delta G_{\mathrm{W} \rightarrow \mathrm{Ol}}$ at multiple resolutions. Other oil/water partitioning free energies have also been proposed for the determination of bead-type assignment in Martini, such as hexadecane, chloroform, and ether. ${ }^{34}$ We restrict ourselves to the water/octanol partitioning free energy because of the difficulty involved in obtaining either experimental partitioning free energy data or predictions for the wide variety of chemistries found in the GDB. The ALOGPS neural network allows us to obtain accurate predictions of $\Delta G_{\mathrm{W} \rightarrow \mathrm{Ol}}$ for new chemistries in this regard. While it is possible that the use of other water/oil partitioning free energies would change the resulting force field parameterizations, previous studies have shown that many Martini partitioning free energies can be viewed as linear transformations of $\Delta G_{\mathrm{W} \rightarrow \mathrm{Ol}}{ }^{29,31,56}$ Therefore, the use of a single type of partitioning free energy should be sufficient as a metric for parameterizing these types of models with respect to the overall hydrophobic/hydrophilic character of the bead types.

Figure 2(e) quantifies the level of information loss using the JSD as the resolution is varied, allowing us to determine how effectively each of these force fields, including Martini, represents CCS projected onto $\Delta G_{\mathrm{W} \rightarrow \mathrm{Ol}}$. The JSD decreases as the number of bead types increase. However, the information retention becomes negligibly greater, essentially plateauing after nine bead types. Remarkably, despite the fact that the Martini force field was parameterized using a small number of chemical compounds (relative to the large distribution of compounds used to parameterize the other models in this work), it shows only a minuscule increase in the JSD. This is mainly due to the lack of a bead type that is placed at $0.0 \mathrm{kcal} / \mathrm{mol}$ on the $\Delta G_{\mathrm{W} \rightarrow \mathrm{Ol}}$ axis. The highly populated peak at this location is the major defining feature for the atomistic distribution of molecules mapping to unimers, and the placement of the Martini Nda and $\mathrm{P} 1$ bead types is insufficient to fully capture this feature. Unfortunately, this increase in the JSD is amplified when comparing the
$\Delta G_{\mathrm{W} \rightarrow \mathrm{Ol}}$ distributions for dimer molecules, whereas for the nine and 16 bead type models, the JSD seems to converge. The combinatorial explosion that results from doubling the size of molecules (i.e., going from unimer to dimer) is reflected in these histograms as a broadening of the total distribution, since more hydrophobic and hydrophilic values of $\Delta G_{\mathrm{W} \rightarrow \mathrm{Ol}}$ are possible as molecule size increases. Figure 2(e) shows that the nine and 16 bead type force fields match this combinatorial explosion.

On the other hand, Figs. 5(e) and 5(f) clearly demonstrate that a high level of accuracy is already achieved with respect to $\Delta G_{\mathrm{W} \rightarrow \mathrm{Ol}}$ using the five-bead-type force field. What, then, is the benefit to using a model with more than five bead types? As shown from Figs. 3 and 4, the main advantage is in back-mapping the coarse-grained representations to their likely atomistic counterparts. ${ }^{30}$ Specifically, the nine bead force field is parameterized not by simply optimizing the JSD, but rather by clustering the GDB molecules into subdistributions based on the type and number of heavy-atom substitutions on the carbon scaffold of each molecule as shown in Fig. 3. As expected, this clustering strategy also results in a minimal value of the JSD, while providing an added convenience. The distributions that were clustered to make this force field provide a method for predicting the chemistries that are most representative of a bead type. Since the standard deviations of these distributions are so large such that some span across three different bead types, this provides only a rough idea of the probable chemistry accessible to a bead type. Moreover, knowledge of the presence of one or two heavy-atom substitutions on a carbon scaffold of up to eight heavy atoms is insufficient for back-mapping given the number of ways in which they can be arranged on that scaffold resulting in wildly different chemical properties. Figure 4 shows how different functional-group pairs will map clearly to specific bead types when the scaffold size is reduced to five heavy atoms. This extra constraint enables a clearer understanding of the range of unique chemistries that are accessible to a specific bead type. Decreasing the size of the scaffold from five to four heavy atoms yields correspondingly narrower distributions of $\Delta G_{\mathrm{W} \rightarrow \mathrm{Ol}}$, meaning that the same functional group pair can be found in fewer bead types. By no longer requiring functional-group pairs and increasing the scaffold size to eight heavy atoms, we begin obtaining distributions similar to those shown in Fig. 3.

Table I also shows that the number of unique functional-group pairs that map to a given bead type decreases as the number of bead types increases, to the point where, for Martini as well as the 16 bead type force field, there exist bead types that essentially backmap to a single functional group pair. Here, we see a clear parallel with structural coarse-graining methods: just as decreasing the size of the beads leads to a finer mapping of the configurational space, increasing the number of bead types leads to a finer mapping of CCS. The efficiency of a coarse-grained model can be optimized by tuning the mapping function and bead size of a coarse-grained model such that the accuracy of the model is balanced with respect to the computational cost of simulating a greater number of particles. By fixing the geometric mapping method and bead size, and only varying the number of bead types possible, we instead balance between the accuracy of representing specific chemical features and the cost of parameterization and validation of the interparticle potentials. We circumvent this cost by interpolating the Martini interaction matrix to obtain accurate parameters for all of the 
force fields presented in this work. However, this cost will be significant for models requiring a more rigorous parameterization scheme relying on other molecular descriptors. Separate from this trade-off between accuracy and parameterization cost, a "screening efficiency" can be defined as the average number of functional-group pairs that map to a single bead type, indicating a larger region of CCS being captured by a single bead type. Unsurprisingly, Table I shows that the five-bead-type force field clearly has the highest screening efficiency.

This statistical analysis of functional-group pairs also suggests a Bayesian approach to computing the probability of a functional group pair, $F$, given a bead type, $T$, represented as $P(F \mid T)$ in Eq. (2). $P(F)$, the Bayesian prior, is the probability of finding the specific functional group pair in the set of molecular fragments (made up of five heavy atoms and containing two functional groups as defined by CHECKMOL) that mapped to single beads, and $P(T)$ is the probability of choosing the given bead type from that same data set. The likelihood, $P(T \mid F)$, shown in the left side of Fig. 4 prescribes the bead type or types to which a fragment could be assigned based on its chemistry-the equivalent of the Martini "bible" for assigning bead types. As shown in Table I, the number of functionalgroup pairs with likelihoods greater than 0.99 (essentially localized to a single bead type) decreases as the number of bead types increases. The Martini force field deviates slightly from this trend, with two more functional-group pairs with high likelihoods as compared to the nine-bead-type force field. This may stem from the parameterization strategy used for Martini that relied on specific molecules and their functional groups rather than aiming to efficiently span chemical space by optimizing the JSD, as proposed in this work. The posterior probabilities, which provide a quantitative description of which chemistries are more representative of each bead type, increase as the number of bead types increases. This effect more easily facilitates the back-mapping of coarse-grained representations. These two quantities, the Bayesian likelihood and posterior, are essential for further exploring CCS covered by specific bead types and enabling both direct and inverse molecular design.

Interestingly, we immediately see a resolution limit with respect to the functional-group pairs that map to specific bead types. Because there are certain length scales on the $\Delta G_{\mathrm{W} \rightarrow \mathrm{Ol}}$ axis that correspond to the distribution of specific functional-group pairs, increasing the number of bead types will naturally split these distributions such that one functional group pair is represented in multiple bead types. Figure 4(a) shows that the majority of functionalgroup pairs are encompassed either by a single bead type or one of its neighbors on the $\Delta G_{\mathrm{W} \rightarrow \mathrm{Ol}}$ axis. Increasing the number of bead types causes these splits to become more exacerbated, spanning multiple bead types for an increased number of functional-group pairs. This is the resolution limit of this type of top-down coarse-graining. The large bead sizes of these models lead to a high degree of variability in the chemistry, meaning that it is no longer obvious which functional group/functional group pair belongs to which bead type. The limit is most evident for the functional-group pairs mapping to the T3 and T13 bead types in Fig. 4(d), indicating that they are placed too close to their neighbors on the $\Delta G_{\mathrm{W} \rightarrow \mathrm{Ol}}$ axis. These functional-group pairs contain some combination of the following functional groups: alkene, alkyne, enamine, hydrazine, hydroxylamine, carboxylic acid derivatives, and fluorine substitution. The placement of these functional groups within a five-carbon scaffold will drastically shift the $\Delta G_{\mathrm{W} \rightarrow \mathrm{Ol}}$ beyond the range of the next-nearest bead type on the $\Delta G_{\mathrm{W} \rightarrow \mathrm{Ol}}$ axis and highlights the limitations of only using this single descriptor for the projection of CCS. While the addition of other partitioning free energies may further increase the accuracy of both the models themselves and the mapping of specific functional groups, these descriptors are encoding essentially the same information as $\Delta G_{\mathrm{W} \rightarrow \mathrm{Ol}}$ : the hydrophobicity of the underlying chemistry. However, determining a suitable orthogonal descriptor and then parameterizing a chemically transferable coarse-grained force field to achieve a more direct relation with CCS is outside the scope of this work, and will be addressed subsequently.

\section{CONCLUSION}

In this work, we use the Jensen-Shannon divergence (JSD) to quantify the information loss in chemically transferable top-down coarse-grained models with varying numbers of bead types, with the GDB as our proxy for chemical compound space (CCS). We find that Martini, while not designed to efficiently reduce CCS, only performs remarkably well in this regard, closely matching the other force fields explicitly designed to minimize the JSD with only a small deviation. All force fields yield roughly the same level of accuracy with respect to $\Delta G_{\mathrm{W} \rightarrow \mathrm{Ol}}$ but vary greatly in their coverage of CCS. We used a Bayesian approach to calculating the probabilities of back-mapping given bead-types to fragments containing specific chemical substitutions. Here, we found it necessary to constrain the size of chemical fragments to five heavy atoms and the presence of two functional groups in order to clearly differentiate between the chemical moieties mapping to each bead type. The results of this Bayesian analysis indicate that increasing the number of bead types decreases the range of accessible chemistry while increasing the corresponding posterior probabilities for each chemistry. However, there is a resolution limit when using this approach, as it does not take into account the specific positions of heteroatom and bond substitutions within a fragment, causing different bead types to appear representative of the same chemistry. Martini, as well as other chemically transferable coarse-grained models, can be used to quickly build structure-property relationships that span broad regions of CCS. Here, we highlight the powerful combination of this method with Bayesian inference, providing an informed mapping of a coarse structure-property relationship to a higher resolution in chemical compound space and further enabling inverse molecular design.

\section{SUPPLEMENTARY MATERIAL}

The attached supplementary material provides details on (i) the changes made to the AUTO-MARTINI code, (ii) systematic errors made by the AUTO-MARTINI algorithm, (iii) the histograms used to calculate JSDs for all force fields described in this work, (iv) statistics for each of the distributions clustered when obtaining the nine-beadtype force field, (v) the parameterization method for the new force fields, and (vi) more information about the functional-group pairs used in the Bayesian analysis. In addition, we provide databases containing the set of GDB compounds mapping to each unimer and dimer, the force field parameters, the trajectories simulated for each 
force field in a repository, the list of all compounds from the NCI database used to construct Fig. 5, the gromacs topology files for the coarse-grained compounds to which the NCI compounds were mapped, and the full list of functional-group pairs identified using CHECKMOL as well as the corresponding probabilities calculated in Fig. 4 for all force fields.

\section{ACKNOWLEDGMENTS}

The authors thank Roberto Menichetti, Joseph F. Rudzinski, and Kurt Kremer for critical reading of this manuscript. The authors acknowledge Igor V. Tetko for an academic research license of the ALOGPS software. The authors thank Mara Jochum for her contributions to the AUTO-MARTIN code. This work was supported by the Emmy Noether program of the Deutsche Forschungsgemeinschaft (DFG) as well as the Max Planck Graduate Center.

\section{REFERENCES}

${ }^{1}$ K. Roy, S. Kar, and R. N. Das, Statistical Methods in QSAR/QSPR (Springer, Cham, 2015), pp. 37-59.

${ }^{2}$ L. Xi, S. Pan, X. Li, Y. Xu, J. Ni, X. Sun, J. Yang, J. Luo, J. Xi, W. Zhu, X. Li, D. Jiang, R. Dronskowski, X. Shi, G. J. Snyder, and W. Zhang, "Discovery of high performance thermoelectric Chalcogenides through reliable high throughput material screening," J. Am. Chem. Soc. 140, 10785-10793 (2018).

${ }^{3}$ A. K. Patel, M. W. Tibbitt, A. D. Celiz, M. C. Davies, R. Langer, C. Denning, M. R. Alexander, and D. G. Anderson, "High throughput screening for discovery of materials that control stem cell fate," Curr. Opin. Solid State Mater. Sci. 20, 202-211 (2016).

${ }^{4}$ N. Mounet, M. Gibertini, P. Schwaller, D. Campi, A. Merkys, A. Marrazzo, T. Sohier, I. E. Castelli, A. Cepellotti, G. Pizzi et al., "Two-dimensional materials from high-throughput computational exfoliation of experimentally known compounds," Nat. Nanotechnol. 13, 246 (2018).

${ }^{5}$ R. Greenaway, V. Santolini, M. Bennison, B. Alston, C. Pugh, M. Little, M. Miklitz, E. Eden-Rump, R. Clowes, A. Shakil et al., "High-throughput discovery of organic cages and catenanes using computational screening fused with robotic synthesis," Nat. Commun. 9, 2849 (2018)

${ }^{6}$ M. C. Burns, J. E. Howes, Q. Sun, A. J. Little, D. V. Camper, J. R. Abbott, J. Phan, T. Lee, A. G. Waterson, O. W. Rossanese et al., "High-throughput screening identifies small molecules that bind to the RAS:SOS:RAS complex and perturb RAS signaling," Anal. Biochem. 548, 44-52 (2018).

${ }^{7}$ C. M. Dobson, "Chemical space and biology," Nature 432, 824-828 (2004).

${ }^{8}$ F. A. Faber, A. S. Christensen, B. Huang, and O. A. von Lilienfeld, "Alchemical and structural distribution based representation for universal quantum machine learning," J. Chem. Phys. 148, 241717 (2018).

${ }^{9}$ M. J. Willatt, F. Musil, and M. Ceriotti, "Feature optimization for atomistic machine learning yields a data-driven construction of the periodic table of the elements," Phys. Chem. Chem. Phys. 20, 29661-29668 (2018).

${ }^{10}$ L. M. Ghiringhelli, J. Vybiral, S. V. Levchenko, C. Draxl, and M. Scheffler, "Big data of materials science: Critical role of the descriptor," Phys. Rev. Lett. 114, 105503 (2015)

${ }^{11}$ R. Ramakrishnan, P. O. Dral, M. Rupp, and O. A. von Lilienfeld, "Big data meets quantum chemistry approximations: The $\delta$-machine learning approach," J. Chem. Theory Comput. 11, 2087-2096 (2015).

${ }^{12}$ K. T. Butler, D. W. Davies, H. Cartwright, O. Isayev, and A. Walsh, "Machine learning for molecular and materials science," Nature 559, 547 (2018).

${ }^{13}$ T. Bereau, R. A. DiStasio, Jr., A. Tkatchenko, and O. A. Von Lilienfeld, "Noncovalent interactions across organic and biological subsets of chemical space: Physics-based potentials parametrized from machine learning," J. Chem. Phys. 148, 241706 (2018).

${ }^{14}$ T. Bereau, D. Andrienko, and K. Kremer, "Research Update: Computational materials discovery in soft matter," APL Mater. 4, 053101 (2016).
${ }^{15} \mathrm{~T}$. Bereau, "Data-driven methods in multiscale modeling of soft matter," in Handbook of Materials Modeling: Methods: Theory and Modeling (Springer, 2018),

pp. 1-12.
${ }^{16}$ W. G. Noid, "Perspective: Coarse-grained models for biomolecular systems," J. Chem. Phys. 139, 090901 (2013).

${ }^{17}$ S. Kmiecik, D. Gront, M. Kolinski, L. Wieteska, A. E. Dawid, and A. Kolinski, "Coarse-grained protein models and their applications," Chem. Rev. 116, 78987936 (2016).

${ }^{18}$ G. A. Voth, Coarse-graining of Condensed Phase and Biomolecular Systems (CRC Press, 2008).

${ }^{19} \mathrm{C}$. Peter and K. Kremer, "Multiscale simulation of soft matter systems-from the atomistic to the coarse-grained level and back," Soft Matter 5, 4357-4366 (2009).

${ }^{20} \mathrm{~W}$. Schommers, "Pair potentials in disordered many-particle systems: A study for liquid gallium," Phys. Rev. A 28, 3599 (1983)

${ }^{21}$ W. Tschöp, K. Kremer, J. Batoulis, T. Bürger, and O. Hahn, "Simulation of polymer melts. I. Coarse-graining procedure for polycarbonates," Acta Polym. 49, 61-74 (1998).

${ }^{22}$ F. Ercolessi and J. B. Adams, "Interatomic potentials from first-principles calculations: The force-matching method," Europhys. Lett. 26, 583-588 (1994).

${ }^{23}$ S. Izvekov and G. A. Voth, "A multiscale coarse-graining method for biomolecular systems," J. Phys. Chem. B 109, 2469-2473 (2005).

${ }^{24}$ G. S. Ayton, W. G. Noid, and G. A. Voth, "Multiscale modeling of biomolecular systems: In serial and in parallel," Curr. Opin. Struct. Biol. 17, 192-198 (2007).

${ }^{25}$ S. J. Marrink, A. H. de Vries, and A. E. Mark, "Coarse grained model for semiquantitative lipid simulations," J. Phys. Chem. B 108, 750-760 (2004).

${ }^{26}$ L. Monticelli, S. K. Kandasamy, X. Periole, R. G. Larson, D. P. Tieleman, and S.-J. Marrink, "The martini coarse-grained force field: Extension to proteins," J. Chem. Theory Comput. 4, 819-834 (2008).

${ }^{27}$ S. J. Marrink and D. P. Tieleman, "Perspective on the martini model," Chem. Soc. Rev. 42, 6801-6822 (2013)

${ }^{28}$ B. M. Bruininks, P. C. Souza, and S. J. Marrink, "A practical view of the martini force field," in Biomolecular Simulations (Springer, 2019), pp. 105-127.

${ }^{29}$ R. Menichetti, K. H. Kanekal, K. Kremer, and T. Bereau, "In silico screening of drug-membrane thermodynamics reveals linear relations between bulk partitioning and the potential of mean force," J. Chem. Phys. 147, 125101 (2017).

${ }^{30}$ R. Menichetti, K. Kremer, and T. Bereau, "Efficient potential of mean force calculation from multiscale simulations: Solute insertion in a lipid membrane," Biochem. Biophys. Res. Commun. 498, 282-287 (2018).

${ }^{31}$ R. Menichetti, K. H. Kanekal, and T. Bereau, "Drug-membrane permeability across chemical space," ACS Cent. Sci. 5, 290-298 (2019).

${ }^{32}$ T. Fink, H. Bruggesser, and J. L. Reymond, "Virtual exploration of the smallmolecule chemical universe below 160 daltons," Angew. Chem., Int. Ed. 44, 15041508 (2005).

${ }^{33}$ T. Fink and J.-L. Reymond, "Virtual exploration of the chemical universe up to 11 atoms of C, N, O, F: Assembly of 26.4 million structures (110.9 million stereoisomers) and analysis for new ring systems, stereochemistry, physicochemical properties, compound classes, and drug discovery," J. Chem. Inf. Model. 47, 342-353 (2007)

${ }^{34}$ S. J. Marrink, H. J. Risselada, S. Yefimov, D. P. Tieleman, and A. H. D. Vries, "The MARTINI force field: Coarse grained model for biomolecular simulations," J. Phys. Chem. B 111, 7812-7824 (2007).

${ }^{35} \mathrm{~T}$. Bereau and K. Kremer, "Automated parametrization of the coarse-grained martini force field for small organic molecules," J. Chem. Theory Comput. 11, 2783-2791 (2015).

${ }^{36}$ I. V. Tetko, V. Y. Tanchuk, and A. E. Villa, "Prediction of n-octanol/water partition coefficients from PHYSPROP database using artificial neural networks and E-state indices," J. Chem. Inf. Comput. Sci. 41, 1407-1421 (2001).

${ }^{37}$ I. V. Tetko and V. Y. Tanchuk, "Application of associative neural networks for prediction of lipophilicity in ALOGPS 2.1 program," J. Chem. Inf. Comput. Sci. 42, 1136-1145 (2002).

${ }^{38}$ T. Bereau, auto_martini master branch, commit: 2c0c095898860cbe086e64b973 b13a45f80137b6, 2019, https://github.com/tbereau/auto martini.

${ }^{39}$ T. E. Oliphant, A Guide to NumPy (Trelgol Publishing, USA, 2006), Vol. 1. 
${ }^{40} \mathrm{~J}$. Lin, "Divergence measures based on the shannon entropy," IEEE Trans. Inf. Theory 37, 145-151 (1991).

${ }^{41}$ A. Chaimovich and M. S. Shell, "Coarse-graining errors and numerical optimization using a relative entropy framework," J. Chem. Phys. 134, 094112 (2011).

${ }^{42}$ T. T. Foley, M. S. Shell, and W. G. Noid, "The impact of resolution upon entropy and information in coarse-grained models," J. Chem. Phys. 143, 243104 (2015).

${ }^{43}$ S. Kullback and R. A. Leibler, "On information and sufficiency," Ann. Math. Stat. 22, 79-86 (1951).

${ }^{44}$ D. J. Wales and J. P. Doye, "Global optimization by basin-hopping and the lowest energy structures of Lennard-Jones clusters containing up to 110 atoms," J. Phys. Chem. A 101, 5111-5116 (1997).

${ }^{45}$ E. Jones, T. Oliphant, and P. Peterson, \{SciPy\}: Open source scientific tools for \{Python\}, 2014.

${ }^{46} \mathrm{~J}$. Nocedal and S. Wright, Numerical Optimization (Springer Science \& Business Media, 2006).

${ }^{47}$ K. H. Kanekal and T. Bereau (2019). "Resolution limit of data-driven coarse-grained models spanning chemical space," Zenodo. http://doi.org/10.5281/ zenodo.3403594.

${ }^{48} \mathrm{~N}$. Haider, "Functionality pattern matching as an efficient complementary structure/reaction search tool: An open-source approach," Molecules 15, 50795092 (2010).
${ }^{49}$ B. Hess, C. Kutzner, D. Van Der Spoel, and E. Lindahl, "GROMACS 4: Algorithms for highly efficient, load-balanced, and scalable molecular simulation," J. Chem. Theory Comput. 4, 435-447 (2008).

${ }^{50} \mathrm{H}$. C. Andersen, "Molecular dynamics simulations at constant pressure and/or temperature,” J. Chem. Phys. 72, 2384-2393 (1980).

${ }^{51}$ C. H. Bennett, "Efficient estimation of free energy differences from Monte Carlo data," J. Comput. Phys. 22, 245-268 (1976).

${ }^{52}$ C. A. López, A. J. Rzepiela, A. H. De Vries, L. Dijkhuizen, P. H. Hünenberger, and S. J. Marrink, "Martini coarse-grained force field: Extension to carbohydrates," J. Chem. Theory Comput. 5, 3195-3210 (2009).

${ }^{53}$ J. H. Voigt, B. Bienfait, S. Wang, and M. C. Nicklaus, "Comparison of the nci open database with seven large chemical structural databases," J. Chem. Inf. Comput. Sci. 41, 702-712 (2001).

${ }^{54}$ R. Alessandri, P. C. Souza, S. Thallmair, M. N. Melo, A. H. de Vries, and S. J. Marrink, "Pitfalls of the Martini Model," J. Chem. Theory Comput. 15, 5448-5460 (2019).

${ }^{55}$ R. Alessandri, J. J. Uusitalo, A. H. de Vries, R. W. Havenith, and S. J. Marrink, "Bulk heterojunction morphologies with atomistic resolution from coarse-grain solvent evaporation simulations," J. Am. Chem. Soc. 139, 3697-3705 (2017).

${ }^{56}$ C. Hoffmann, R. Menichetti, K. H. Kanekal, and T. Bereau, "Controlled exploration of chemical space by machine learning of coarse-grained representations," Phys. Rev. E 100, 033302 (2019). 